\title{
Genetic Analysis of Leucine Content in Indica-Japonica Hybrid Rice (Oryza sativa L.)
}

\author{
Xiaoming Zhang ${ }^{1,2}$, Chunhai Shi ${ }^{2 *}$, Jianguo $\mathrm{Wu}^{2}$, Shenghai Ye ${ }^{1}$, Genliang Bao ${ }^{1}$, Wenchao Yan ${ }^{1}$ \\ ${ }^{1}$ Institute of Crop Research and Atomic Energy Utilization, Zhejiang Academy of Agricultural Sciences, Hangzhou, People’s Repub- \\ lic of China; ${ }^{2}$ College of Agriculture and Biotechnology, Zhejiang University, Hangzhou, People’s Republic of China. \\ Email: chhshi@zju.edu.cn
}

Received August $10^{\text {th }}, 2010$; revised September $6^{\text {th }}, 2010$; accepted September $13^{\text {th }}, 2010$

\begin{abstract}
Genetic control of leucine content in indica-japonica hybrid rice (Oryza sativa L.) was studied in 35 crosses of $F_{1}$ and $F_{2}$ generations, which were derived from crossing 7 male sterile indica rice lines with 5 restorer japonica rice lines along with their parents. Two genetic models and their corresponding statistical methods for quantitative traits of triploid seeds in cereal crops were used for the analysis. The first was the unconditional genetic model, which refers to the analysis of cumulative measurements (from flowering to a specific time) along the developmental stages, while the second was the conditional genetic model, which relates to analysis from one developmental stage to another stage $(\boldsymbol{t}-1 \rightarrow$ $\boldsymbol{t})$. The results showed that leucine content of indica-japonica hybrid rice was controlled by the expression of triploid endosperm effect (endosperm additive effect and endosperm dominant effect), cytoplasm effect, diploid maternal plant effect (maternal additive effect and maternal dominant effect) and their environmental interaction effects. Of these effects, endosperm dominant effect and maternal dominant effect were more important at the earlier stages, while endosperm additive effect and maternal additive effect were more important at the later stages of rice grain development under both unconditional and conditional genetic analyses. Due to the high heritabilities, which came from endosperm, maternal and cytoplasm effects for leucine content at different developmental stages, selection for leucine content of indica-japonica hybrid rice would be more efficient at early generations in breeding programs.
\end{abstract}

Keywords: Indica-Japonica Hybrid Rice, Developmental Genetics, Quality, Leucine Content, Genetic Variance, Conditional Genetic Variance, Heritability

\section{Introduction}

The use of indica-japonica $\mathrm{F}_{1}$ hybrids offers a way of combining the best attributes of both types. Advantages of these hybrids include strong tillering ability, thick culms and larger panicles. However, some disadvantages have also been noted, such as longer growing duration, greater plant height and reduced spikelet fertility. Recently, significant progress has been made in improving agronomic traits in an indica-japonica hybrid breeding project [1] and breeders are now paying greater attention to improving rice grain quality traits.

In most reports, phenotypic values measured at maturity are used when analyzing rice quality traits [2-7], but this does not provide information on the developmental processes of these traits. Although recent quantitative trait analysis software makes developmental genetic analysis possible [8], the literatures are limited

*corresponding author to indica rice apparent quality and milling quality [9-11]. A little information on nutrition of indica-japonica hybrid rice has been reported, especially for amino acids. One reason for the lack of information is the difficulty in obtaining a significant quantity of $F_{1}$ seeds for analysis due to incompatibility between the indica and japonica rice subspecies when crossed. Some japonica wide compatible restorers developed by our projects make this genetic analysis in indica-japonica hybrid rice possible.

Leucine $\left(\mathrm{C}_{6} \mathrm{H}_{13} \mathrm{NO}_{2}\right)$ is a neutral, genetically coded essential amino acid in human nutrition, which is very important in human health. The expression of genes for leucine content at different grain-filling stages is yet to be understood. Understanding the dynamics of gene expression for leucine content in contrasting environments may prove helpful for future improvements in the nutritional quality of rice. The objective of this study was to clarify the developmental behavior of leucine content 
gene expression in indica-japonica hybrid rice. The developmental dynamic expression mechanism and the heritability components from different genetic systems were estimated by unconditional and conditional genetic models.

\section{Materials and Methods}

\subsection{Plant Material}

Seven indica cytoplasmic male sterile lines (A lines) (Zhe 38, Xieqingzao, K17, Zhenshan 97, Zhenong 8010, Jin 23 and II -32) and their maintainers (B lines), and 5 japonica wide compatible restorers ( $\mathrm{R}$ lines) ( $\mathrm{T}$ 748, $\mathrm{T}$ 42, $\mathrm{R}$ 1252, Linhui 422 and Zhong 9308) with significant variation in leucine contents were used in this experiment (Table 1).

\subsection{Field Experiment}

$F_{1}$ seeds were obtained by crossing female A lines to male $\mathrm{R}$ lines $(\mathrm{A} \times \mathrm{R})$ using a factorial design. The $\mathrm{F}_{1} \mathrm{~s}$ and their parents were sown at the experimental farm, Zhejiang Academy of Agricultural Sciences, China. After 25 days, seedlings were individually transplanted at a spacing of $20 \times 26 \mathrm{~cm}$. There were 36 plants in each plot and two replications. A second experiment was conducted 7 months later using the same methods to provide contrasting environmental conditions at Hainan province, China. Seeds from parental lines and $\mathrm{F}_{2}$ seeds from $\mathrm{F}_{1}$ plants were collected at 7-, 14-, 21-, 28- and 35-day after flowering from the central 16 plants within each plot. The $F_{1}$ plants resulted from $\mathrm{A} \times \mathrm{R}$ crosses. The grain-filling period was devided into stages, the initial stage (1-7days after flowering), early stage (8-14days), middle stage (15-21days), late stage (22-28 days) and ripe stage (29-35days).

\subsection{Leucine Content Analysis}

All seed hulls of $F_{1} s, F_{2} s$ and their parents were removed using a Yanmar ST50 dehuller from Japan, then milled using a sample miller (model JB-20, Zhejiang province, China). Samples were further ground to 100 mesh with a cyclone grinder (model 3010-019, Fort Collins, Colorado, USA). Leucine content was determined using the amino acid analysis method described by Wu et al. [12] with two replications for each sample.

\subsection{Statistical Method}

Two developmental genetic models, the unconditional genetic model [13] and the conditional genetic model [8], for quantitative traits of endosperm in cereal crops were used to estimate the variance components for genetic main effects and genotype- environmental interaction effects at different grain-filling stages. For the unconditional genetic analysis, which refers to the analysis of cumulative measurements through progressive developmental stages, the genetic effects were defined as accumulated effects of genes expressed from flowering (0) to a particular time $(\boldsymbol{t})$. The variance components were divided into endosperm additive variance $\left(V_{A}\right)$, endosperm dominance variance $\left(V_{D}\right)$, cytoplasmic variance $\left(V_{C}\right)$, maternal additive variance $\left(V_{A m}\right)$, maternal

Table 1. Leucine content (\%) of 12 parents at five different developmental stages.

\begin{tabular}{|c|c|c|c|c|c|}
\hline Parent & Initial stage & Early stage & Middle stage & Late stage & Ripening stage \\
\hline \multicolumn{6}{|l|}{ Female group } \\
\hline Zhe38 & 1.113a & $1.122 \mathrm{a}$ & $1.274 \mathrm{a}$ & 1.203ab & 1.083ab \\
\hline Xieqingzao & $0.769 b$ & $0.805 d$ & $0.921 \mathrm{~cd}$ & $0.926 \mathrm{bc}$ & $0.781 \mathrm{e}$ \\
\hline K17 & $0.788 b$ & $0.751 d$ & $0.906 \mathrm{~cd}$ & $0.934 \mathrm{bc}$ & 0.867 cde \\
\hline Zhenshan97 & $0.984 \mathrm{ab}$ & $0.966 \mathrm{bc}$ & 1.097abc & 1.198ab & $0.951 \mathrm{bcd}$ \\
\hline Zhenong8010 & $0.738 b$ & $0.872 \mathrm{~cd}$ & $0.849 d$ & $1.021 \mathrm{abc}$ & $0.743 e$ \\
\hline $\operatorname{Jin} 23$ & 1.008ab & 1.019ab & $1.186 \mathrm{ab}$ & $1.271 \mathrm{a}$ & $1.120 \mathrm{a}$ \\
\hline II-32 & $0.723 b$ & $0.757 d$ & $0.860 \mathrm{~cd}$ & $0.991 \mathrm{abc}$ & 0.891 cde \\
\hline Standard deviation & 0.156 & 0.142 & 0.171 & 0.143 & 0.142 \\
\hline \multicolumn{6}{|l|}{ Male group } \\
\hline $\mathrm{T} 42$ & 1.029ab & $1.041 \mathrm{ab}$ & 1.070abcd & $1.080 \mathrm{abc}$ & $0.959 \mathrm{bc}$ \\
\hline R1252 & $0.852 \mathrm{ab}$ & $0.829 d$ & $0.947 \mathrm{bcd}$ & $1.024 \mathrm{abc}$ & 0.824 cde \\
\hline Linhui422 & $0.871 \mathrm{ab}$ & $0.820 \mathrm{~d}$ & $0.935 \mathrm{~cd}$ & $0.940 \mathrm{bc}$ & $0.807 \mathrm{de}$ \\
\hline Zhong9308 & $0.734 b$ & $0.771 d$ & $0.838 d$ & $0.863 c$ & $0.957 \mathrm{bcd}$ \\
\hline Standard deviation & 0.119 & 0.104 & 0.096 & 0.094 & 0.073 \\
\hline
\end{tabular}

a,b,c,d,e denote significance at 0.05 probability level. 
dominance variance $\left(V_{D m}\right)$, and their endosperm additive $\times$ environment interaction variance $\left(V_{A E}\right)$, endosperm dominance interaction variance $\left(V_{D E}\right)$, cytoplasm interaction variance $\left(V_{C E}\right)$, maternal additive interaction variance $\left(V_{A m E}\right)$ and maternal dominance interaction variance $\left(V_{D m E}\right)$, and residual variance $\left(V_{e}\right)$. Since some endosperm genes were derived from maternal plants, there were possible covariances between endosperm effects and maternal effects including additive covariance $C_{A . A m}$, dominance covariance $C_{D . D m}$, additive interaction covariance $C_{A E . A m E}$, and dominance interaction covariance $C_{D E . D m E}$. The partitioning for the phenotypic variance $\left(V_{P(t)}\right)$ was:

$$
\begin{aligned}
V_{P}= & V_{A}+V_{D}+V_{C}+V_{A m}+V_{D m}+V_{A E}+V_{D E}+V_{C E}+V_{A m E}+V_{D m E} \\
& +2\left(C_{A \cdot A m}+C_{D \cdot D m}\right)+2\left(C_{A E \cdot A m E}+C_{D E \cdot D m E}\right)+V_{e}
\end{aligned}
$$

For the conditional genetic analysis, which refers to grain analysis within each stage, the conditional developmental genetic models could be used to estimate conditional variances during rice grain filling periods $(\boldsymbol{t}-1 \rightarrow \boldsymbol{t})$ for leucine content. These conditional variance components were $V_{A(t \mid t-1)}$ (conditional endosperm additive variance), $V_{D(t \mid t-1)}$ (conditional endosperm dominance variance), $V_{C(t \mid t-1)}$ (conditional cytoplasmic variance), $V_{A m(t \mid t-1)}$ (conditional maternal additive variance), $V_{D m(t \mid t-1)}$ (conditional maternal dominance variance), $V_{A E(t \mid t-1)}$ (conditional endosperm additive interaction variance), $V_{D E(t \mid t-1)}$ (conditional endosperm dominance interaction variance), $V_{C E(t \mid t-1)}$ (conditional cytoplasm interaction variance), $V_{A m E(t \mid t-1)}$ (conditional maternal additive interaction variance), $V_{D m E(t \mid t-1)}$ (conditional maternal dominance interaction variance), $C_{A . A m(t \mid t-1)}$ (conditional additive covariance), $C_{D . D m(t \mid t-1)}$ (conditional dominance covariance), $C_{A E . A m E(t \mid t-1)}$ (conditional additive interaction covariance), $C_{D E . D m E(t \mid t-1)}$ (conditional dominance interaction covariance) and $\mathrm{Ve}_{(t \mid t-1)}$ (conditional residual variance). The partitioning for the conditional phenotypic variance $\left(V_{P(t \mid t-1)}\right)$ was:

$$
\begin{aligned}
V_{P(t \mid t-1)}= & V_{A(t \mid t-1)}+V_{D(t \mid t-1)}+V_{C(t \mid t-1)}+V_{A m(t \mid t-1)}+V_{D m(t \mid t-1)} \\
& +V_{A E(t \mid t-1)}+V_{D E(t \mid t-1)}+V_{C E(t \mid t-1)}+V_{A m E(t \mid t-1)} \\
& +V_{D m E(t \mid t-1)}+2\left(C_{A \cdot A m(t \mid t-1)}+C_{D \cdot D m(t \mid t-1)}\right) \\
& +2\left(C_{A E \cdot A m E(t \mid t-1)}+C_{D E \cdot D m E(t \mid t-1)}\right)+V_{e(t \mid t-1)}
\end{aligned}
$$

Heritabilities $\left(h^{2}\right)$ can be further divided into general heritability $\left(h_{G}^{2}\right)$ and environment interaction heritability $\left(h_{G E}^{2}\right)$ by unconditional genetic analysis. The general heritability $\left(h_{\mathrm{G}}^{2}\right)$, which was controlled by genetic main effects, could be further divided into endosperm general heritability $\left(h_{G o}^{2}=\left(V_{A}+C_{A \cdot A m}\right) / V_{P}\right)$, cytoplasm heritability $\left(h_{G c}^{2}=V_{C} / V_{P}\right)$ and maternal general heritability $\left(h_{G m}^{2}\right.$ $\left.=\left(V_{A m}+C_{A \cdot A m}\right) / V_{P}\right)$. The interaction heritability $\left(h_{G E}^{2}\right)$, which was controlled by genotype $\times$ environmental interaction effects, also could further be divided into endosperm $\times$ environment interaction heritability $\left(h_{G o E}^{2}=\left(V_{A E}\right.\right.$ $\left.\left.+C_{A E \cdot A m E}\right) / V_{P}\right)$, cytoplasmic $\times$ environment interaction heritability $\left(h_{G c E}^{2}=V_{C E} / V_{P}\right)$ and maternal $\times$ environment interaction heritability $\left(h_{G m E}^{2}=\left(V_{A m E}+C_{A E \cdot A m E}\right) / V_{P}\right)$. The partitioning for the total narrow-sense heritability was:

$$
h^{2}=h_{G}^{2}+h_{G E}^{2}=\left(h_{G o}^{2}+h_{G c}^{2}+h_{G m}^{2}\right)+\left(h_{G o E}^{2}+h_{G c E}^{2}+h_{G m E}^{2}\right)
$$

The Jackknife re-sampling method was used by sampling generation means of entries for estimating standard errors of variances components, covariance and heritabilities [14-15].

\section{Results}

\subsection{Difference of Leucine Content in Parents and their Descendants}

There was significant variation for leucine content both in female parents and in male parents at five different filling stages (Table 1). Of the A lines, Zhe38 and Jin23 had high leucine content at the initial stage, while of the $\mathrm{B}$ lines, T42 had the highest leucine content at the initial stage. Zhenong 8010 and T748 were developed quickly than others at early filling stage, Xieqingzao, Zhenshan97, R1252 and Linhui422 were developed faster at middle filling stage. All the parents except Zhong 9318 reached their highest level of leucine content at the late grain-filling stage, and the results showed that Jin23 was the highest (1.271\%) and K17 was the lowest (0.934\%). At the ripe stage, leucine content of Jin 23 was significant high than that of II-32, K17, T748, R1252, Linhui 422, Xieqingzao and Zhenong 8010 (Table 1).

The mean leucine contents of indica-japonica hybrids of females, males and their $\mathrm{F}_{1}$ and $\mathrm{F}_{2}$ descendants are shown in Figure 1. There were continuous increases after

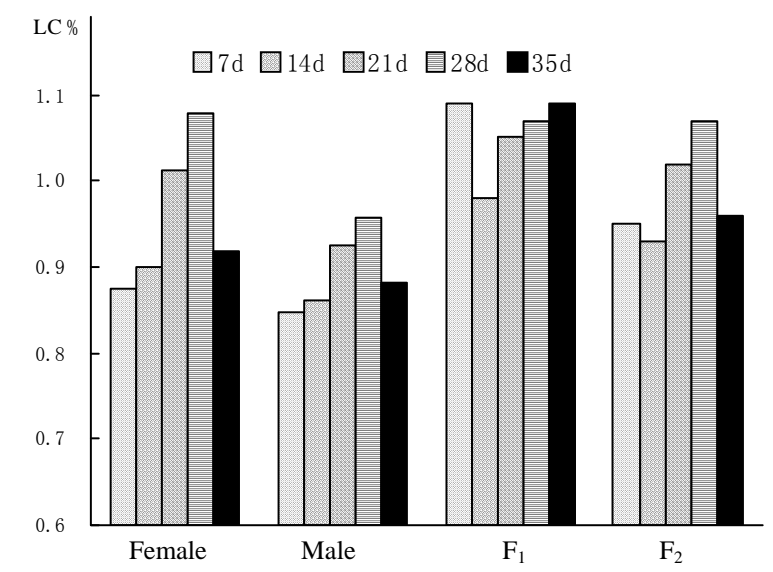

Figure 1. Leucine contents of $\mathbf{4}$ generations at five different filling stages. 
flowering until the late stage and the mean values for these four stages were $0.858,0.899,1.013$ and $1.078 \%$ for females, and $0.844,0.865,0.925$ and $0.958 \%$ for males, respectively. Leucine content decreased to 0.920 and $0.890 \%$ at the ripe stage, respectively. At all grain-filling stages leucine content of female parents was higher than that of the male parents. In both groups of females and males, the highest leucine content level was observed at the late developmental stage. The leucine contents of $\mathrm{F}_{1} \mathrm{~S}$ were $1.094,0.978,1.052,1.073$ and $1.092 \%$, while those of $F_{2}$ were $0.947,0.926,1.022,1.070$ and $0.960 \%$, respectively. Heterosis for leucine content was observed in both $F_{1}$ and $F_{2}$, but $F_{1}$ heterosis was stronger than $F_{2}$, due to the segregation among $F_{2}$ plants.

\subsection{Unconditional Genetic Analysis for Leucine Content at Different Filling Stages}

Table 2 shows parameters generated by the unconditional genetic model of developmental genetics and corresponding statistical analysis for quantitative trait cumulative effects in cereal crops [13]. This analysis showed that leucine content of indica-japonica hybrid rice was controlled by genetic main effects as well as by genotype $x$ environmental interaction effects at five different filling stages. Compared with genetic main effects $\left(V_{\mathrm{G}}=V_{\mathrm{A}}\right.$ $+V_{D}+V_{C}+V_{A m}+V_{D m}$ ), leucine content was mainly controlled by genotype $\times$ environmental interact- ion effects $\left(V_{G E}=V_{A E}+V_{D E}+V_{C E}+V_{A m E}+V_{D m E}\right)$ at $7^{\text {th }}, 14^{\text {th }}, 21^{\text {st }}$ and $35^{\text {th }}$ day after flowering and the $V_{\mathrm{GE}}$ were accounted for $74.87,93.44,93.79$ and $61.23 \%$ of total genetic vari- ance $\left(V_{G E} /\left(V_{G}+V_{G E}\right)\right)$, respectively. However, the fourth developmental stage (late stage, $28^{\text {th }}$ day after flowering) was mainly controlled by the genetic main effect. In summary, leucine content was controlled by both genetic main effects and their genotype $\times$ environmental interaction effects at all filling stages, but was mainly controlled by their interaction effects in all but the late stage.

Among genetic main effects, there were endosperm dominant effect and maternal additive effect at the initial stage, maternal dominant effect at early stage, endosperm dominant effect at middle stage, endosperm additive effect, cytoplasmic effect, maternal additive effect and maternal dominant effect at late stage, and endosperm additive effect, endosperm dominant effect and maternal additive effect at ripe stage. All genotype $\times$ environmental interaction effects were significant, except for the additive interaction effects at middle stage and additive interaction effect and maternal additive interaction effects at late stage.

Table 2 also showed that leucine content was mainly controlled by additive effects both in genetic main effect and in their genotype $\times$ environmental interaction effects. It accounted for $97.89 \%\left(\left(V_{A}+V_{A m}\right) /\left(V_{A}+V_{A m}+V_{C}+\right.\right.$ $\left.\left.V_{D}+V_{D m}\right)\right)$ and 60.91\% $\left(\left(V_{A E}+V_{A m E}\right) /\left(V_{A E}+V_{A m E}+V_{C E}\right.\right.$ $\left.\left.+V_{D E}+V_{D m E}\right)\right)$ of the variance at ripe stage, respectively. This means that selection will be effective in early generations because additive effects can be fixed during subsequent inbreeding. There was no relationship detected between the expression of endosperm and maternal genes

Table 2. Estimates of unconditional variance components at different developmental stages for leucine content in indica-japonica rice.

\begin{tabular}{|c|c|c|c|c|c|c|c|c|c|c|}
\hline \multirow{3}{*}{$\begin{array}{c}\text { Parameter } \\
V_{A}\end{array}$} & \multicolumn{10}{|c|}{ Developmental time (days after flowering) } \\
\hline & \multicolumn{2}{|c|}{$7 \mathrm{~d}$} & \multicolumn{2}{|c|}{$14 \mathrm{~d}$} & \multicolumn{2}{|c|}{$21 \mathrm{~d}$} & \multicolumn{2}{|c|}{$28 \mathrm{~d}$} & \multicolumn{2}{|c|}{$35 \mathrm{~d}$} \\
\hline & 0.000 & & 0.000 & & 0.000 & & 7.710 & $* *$ & 12.830 & $* *$ \\
\hline$V_{D}$ & 2.597 & $* *$ & 0.000 & & 1.841 & $* *$ & 0.000 & & 0.286 & $* *$ \\
\hline$V_{C}$ & 0.000 & & 0.000 & & 0.000 & & 6.839 & $* *$ & 0.000 & \\
\hline$V_{A m}$ & 11.825 & $* *$ & 0.000 & & 0.000 & & 13.169 & $* *$ & 13.000 & ** \\
\hline$V_{D m}$ & 0.000 & & 3.509 & $* *$ & 0.000 & & 2.029 & $* *$ & 0.000 & \\
\hline$V_{A E}$ & 14.306 & $* *$ & 12.496 & $* *$ & 0.000 & & 0.000 & & 9.037 & $* *$ \\
\hline$V_{D E}$ & 3.223 & $* *$ & 3.086 & $* *$ & 2.282 & $* *$ & 2.587 & $* *$ & 3.440 & ** \\
\hline$V_{C E}$ & 6.346 & $* *$ & 8.549 & $* *$ & 9.940 & $* *$ & 4.032 & $* *$ & 8.416 & $* *$ \\
\hline$V_{A m E}$ & 14.762 & $* *$ & 21.192 & $* *$ & 12.082 & $* *$ & 0.000 & & 16.079 & ** \\
\hline$V_{D m E}$ & 4.330 & $* *$ & 4.689 & $* *$ & 3.516 & $* *$ & 3.042 & $* *$ & 4.265 & $* *$ \\
\hline$C_{A \cdot A m}$ & 0.000 & & 0.000 & & 0.000 & & -4.559 & & -2.177 & \\
\hline$C_{D \cdot D m}$ & 0.000 & & 0.000 & & 0.000 & & 0.000 & & 0.000 & \\
\hline$C_{A E \cdot A m E}$ & 1.695 & & -10.410 & & 0.000 & & 0.000 & & 2.263 & \\
\hline$C_{D E \cdot D m E}$ & -0.650 & & 0.011 & & 0.028 & & -0.330 & & -0.059 & \\
\hline$V_{e}$ & 0.440 & $* *$ & 0.668 & $* *$ & 0.553 & $* *$ & 0.467 & $* *$ & 0.272 & $* *$ \\
\hline
\end{tabular}

** significant at 0.01 probability level. 
since the genetic main covariances $\left(C_{A . A m}\right.$ or $\left.C_{D . D m}\right)$ and genotype $\times$ environmental interaction covariances $\left(C_{A E \cdot A m E}\right.$ or $C_{D E \cdot D m E}$ ) between the endosperm and maternal effects were not significant. Leucine content may be influenced by sampling errors according to the significant residual variances $\left(V_{e}\right)$. But, in comparison with other genetic parameters, the values of $V_{e}$ were lower.

\subsection{Conditional Genetic Analysis for Leucine Content at Different Filling Stages}

The genetic variances estimated by unconditional genetic analysis revealed accumulated genetic effects which were expressed from flowering to time $\boldsymbol{t}(\mathbf{0} \rightarrow \boldsymbol{t})$, and the results could not clarify gene expression at each special developmental stage $(t-1 \rightarrow t)$. Therefore, conditional genetic analysis was used to clearly explain the dynamic gene expression at each filling stage $(\boldsymbol{t}-1 \rightarrow \boldsymbol{t})$.

Table 3 shows the parameters generated by the conditional genetic model of developmental genetics and corresponding statistical analysis for quantitative trait periodical effects of triploid in cereal crops [8]. There was no detection of new gene expression for conditional endosperm additive main effects $\left(V_{A(7 \mid 0),} V_{A(14 \mid 7)}\right.$ and $V_{A}$ (21|14) from initial- to middle-filling stages, conditional maternal additive main effects $\left(V_{A m(14 \mid 7)}\right.$ and $\left.V_{A m(21 \mid 14)}\right)$ at early- and middle-filling stages, conditional endosperm dominant main effects $\left(V_{D(21 \mid 14)}\right.$ and $\left.V_{D(28 \mid 21)}\right)$ at middle- and late-stages, conditional maternal dominant main effects $\left(V_{D m(7 \mid 0)}\right.$ and $\left.V_{D m(28 \mid 21)}\right)$ at initial- and late-filling stages and conditional cytoplasmic main effects $\left(V_{D m(7 \mid 0)}\right.$ and $\left.V_{C(35 \mid 28)}\right)$ at initial- and ripe stages, since the conditional genetic main variances at these filling stages were not significant in this experiment. The unconditional maternal dominant main effect at late filling stage in Table 2 might be due to the dominant effect expressed at the former stage, i.e. at middle stage (14-21 days after flowering), and then continual expression from activated genes at this stage. Similarly, the endosperm dominant main effect was significant at 21 days after flowering by unconditional genetic analysis, but was not significant using conditional genetic analysis. In contrast, conditional maternal dominant effects at the ripe stage were found by conditional genetic analysis, but not found by unconditional genetic analysis in Table 2. This may reflect interruption of gene expression during different filling stages or the gradual activation of quantitative genes through the filling period(s). It may also indicate that there were differences in regulation of gene expression in triploid endosperm, cytoplasm and diploid maternal plant genetic systems. These results were not detected by the unconditional genetic variance analysis.

For the conditional interaction analysis, new genetic effects from gene expression was not detected for the con-

Table 3. Estimates of conditional variance components for leucine content at different developmental stages in indica-japonica rice.

\begin{tabular}{|c|c|c|c|c|c|c|c|c|c|c|}
\hline \multirow{3}{*}{$\begin{array}{c}\text { Parameter } \\
V_{A(t \mid t-1)}\end{array}$} & \multicolumn{10}{|c|}{ Developmental stage of grain $(\mathrm{t} \mid \mathrm{t}-1)$} \\
\hline & \multicolumn{2}{|c|}{$7 \mathrm{~d} \mid 0 \mathrm{~d}$} & \multicolumn{2}{|c|}{$14 \mathrm{~d} \mid 7 \mathrm{~d}$} & \multicolumn{2}{|c|}{ 21d|14d } & \multicolumn{2}{|c|}{ 28d|21d } & \multicolumn{2}{|c|}{ 35d|28d } \\
\hline & 0.000 & & 0.000 & & 0.000 & & 4.324 & $* *$ & 9.703 & $* *$ \\
\hline$V_{D(t \mid t-1)}$ & 2.597 & $* *$ & 4.700 & $* *$ & 0.000 & & 0.000 & & 1.279 & $* *$ \\
\hline$V_{C(t \mid t-1)}$ & 0.000 & & 7.137 & $* *$ & 3.621 & $* *$ & 2.848 & $* *$ & 0.000 & \\
\hline$V_{A m(t \mid t-1)}$ & 11.825 & $* *$ & 0.000 & & 0.000 & & 9.158 & $* *$ & 6.559 & $* *$ \\
\hline$V_{D m(t \mid t-1)}$ & 0.000 & & 3.277 & $* *$ & 6.975 & $* *$ & 0.000 & & 1.076 & $* *$ \\
\hline$V_{A E(t \mid t-1)}$ & 14.306 & $* *$ & 13.463 & $* *$ & 10.171 & $* *$ & 0.000 & & 8.072 & $* *$ \\
\hline$V_{D E(t \mid t-1)}$ & 3.223 & $* *$ & 0.000 & & 4.543 & $* *$ & 2.809 & $* *$ & 2.503 & $* *$ \\
\hline$V_{C E(t \mid t-1)}$ & 6.346 & $* *$ & 0.000 & & 0.000 & & 0.000 & & 0.000 & \\
\hline$V_{A m E(t \mid t-1)}$ & 14.762 & $* *$ & 7.358 & $* *$ & 3.852 & $* *$ & 0.000 & & 11.931 & $* *$ \\
\hline$V_{D m E(t \mid t-1)}$ & 4.330 & $* *$ & 6.009 & $* *$ & 0.000 & & 2.290 & $* *$ & 3.941 & $* *$ \\
\hline$C_{A \cdot A m(t \mid t-1)}$ & 0.000 & & 0.000 & & 0.000 & & -2.819 & & -8.654 & \\
\hline$C_{D \cdot D m(t \mid t-1)}$ & 0.000 & & -0.080 & & 0.000 & & 0.000 & & 3.889 & $* *$ \\
\hline$C_{A E \cdot A m E(t \mid t-1)}$ & 1.695 & & -46.510 & & -2.301 & & 0.000 & & 2.404 & \\
\hline$C_{D E \cdot D m E(t \mid t-1)}$ & -0.650 & & 0.000 & & 0.000 & & -0.436 & & -0.290 & \\
\hline$V_{e(t \mid t-1)}$ & 0.440 & $* *$ & 0.738 & $* *$ & 0.794 & $* *$ & 0.413 & $* *$ & 0.257 & $* *$ \\
\hline
\end{tabular}

** was significant at 0.01 probability level. 
ditional endosperm additive interaction effect $\left(V_{A E}\right.$ (28|21)), conditional maternal additive interaction effect $\left(V_{A m E ~(28 \mid 21)}\right)$ at late filling stage, conditional endosperm domin- ant interaction effect $\left(V_{D E(14 \mid 7)}\right)$ at early stage, conditional maternal dominant interaction effect $\left(V_{D m E}\right.$ (21|14) $)$ at middle stage and conditional cytoplasm interaction effect $\left(V_{C E \text { (14|7) }}, V_{C E(21 \mid 14)}, V_{C E \text { (28|21) }}\right.$ and $\left.V_{C E \text { (35|28) }}\right)$ at early-, middle- and ripe-stage. The $V_{D E}$ at early stage, $V_{D m E}$ at middle stage and $V_{C E}$ at early-, middle- and ripe-stage were significant using the unconditional genetic analysis in Table 2, but were not significant using the conditional genetic analysis in Table 3 . It is likely that there was continual expression of activated genes at these stages.

There was significant positive conditional dominant interaction covariance $\left(C_{D \cdot D m(35128)}=3.889 * *\right)$ at ripe stage, which showed that the new expression for dominance effect from endosperm nuclear genes was closely related with that from maternal nuclear genes at the ripe stage. Significant conditional residual variances $\left(V_{e(t)-1)}\right)$ showed that new expression of genes for leucine content at the ripe stage could be influenced by sampling errors, but these values were lower than the genetic variances.

\subsection{Estimation of Heritability at Different Filling Stages}

The results in Table 4 showed that general heritability components from cytoplasm and endosperm were not significant at first three stages, but were significant at the next two developmental stages for endosperm and the fourth stage for cytoplasm, while their interaction heritability components were all significant except for endosperm interaction heritabilities at middle- and late-stage. Maternal general heritabilities were significant except for early- and middle-stage and its interaction heritabilities except for late filling stage. The interaction heritabilities including endosperm, maternal and cytoplasm interaction heritabilities were larger than their general heritability components at the first three stages and the last filling st- age, i.e. initial-, early-, middle- and ripe stage, while general heritability was larger than the interaction heritability at late filling stage. The total narrow sense heritability values $\left(h^{2}\right)$ at most filling stages for leucine content were over $75 \%\left(h_{(7 d)}=84.50, h_{(14 d)}=64.14, h_{(21 d)}=\right.$ $72.75, h_{(28 d)}=75.20$ and $\left.h_{(35 d)}=87.97 \%\right)$. The general heritabilities were 19.70, 0.00, 0.00, 61.80 and 31.73\% and interaction heritabilities were 64.80, 64.14, 72.75, 13.40 and $56.23 \%$, respectively. With regard to the components of heritability, maternal and cytoplasm general heritabilities and interaction heritabilities were more important for leucine content $\left(h_{G m}^{2}+h_{G c}^{2}+h_{G m E}^{2}+h_{G c E}^{2}=\right.$ $57.80,57.89,72.75,75.20$ and $55.53 \%$, respectively) in this experiment. These data suggest that improving leucine content would be more efficient when selection is based on maternal plants in early indica-japonica generations.

\section{Discussion}

The genetic behavior of rice quantitative traits is simultaneously controlled by endosperm, maternal and cytoplasm effects. Genes controlling the performance of complex quantitative traits, were expressed at various times during different developmental stages [8-10]. Analysis for quantitative traits has been gradually emphasized in developmental genetics.

This research revealed that gene expression varied during most filling stages in endosperm, cytoplasm and maternal genetic systems across environments. Leucine content was mainly controlled by additive effects, maternal effects and cytoplasmic effects, both by variance analysis and by heritabilities analysis. This means that selection for leucine content would be more efficient at early generations in a rice breeding program. The results of conditional genetic variance analysis for leucine content further indicated that there was a phenomenon of interval expression for some genes among rice filling stages. For example, the conditional genetic analysis, found a significant maternal additive effect at the initial stage, which was non-significant at the early- and middle- stage, but

Table 4. Estimates of heritability components (\%) for leucine content at different developmental stages in indica-japonica rice.

\begin{tabular}{|c|c|c|c|c|c|c|c|c|c|c|}
\hline \multirow{2}{*}{ Parameter } & \multicolumn{10}{|c|}{ Heritability } \\
\hline & \multicolumn{2}{|c|}{$7 d$} & \multicolumn{2}{|c|}{$14 d$} & \multicolumn{2}{|c|}{$21 d$} & \multicolumn{2}{|c|}{$28 \mathrm{~d}$} & \multicolumn{2}{|c|}{$35 d$} \\
\hline$h_{G o}^{2}$ & 0.000 & & 0.000 & & 0.000 & & 10.470 & & 15.740 & \\
\hline$h_{G c}^{2}$ & 0.000 & & 0.000 & & 0.000 & & 22.724 & $* *$ & 0.000 & \\
\hline$h_{G m}^{2}$ & 19.700 & $* *$ & 0.000 & & 0.000 & & 28.608 & $* *$ & 15.992 & + \\
\hline$h_{G o E}^{2}$ & 26.700 & & 6.249 & & 0.000 & & 0.000 & & 16.697 & $*$ \\
\hline$h_{G c E}^{2}$ & 10.600 & & 25.603 & $* *$ & 32.837 & $* *$ & 13.397 & $* *$ & 12.435 & $*$ \\
\hline$h_{G m E}^{2}$ & 27.500 & & 32.289 & $* *$ & 39.914 & $* *$ & 0.000 & & 27.101 & $* *$ \\
\hline
\end{tabular}

,$+ *$ and $* *$ denote significance at $0.10,0.05$ and 0.01 probability levels, respectively. 
was again significant at the late- and ripe- stage.

Genotype $\times$ environment interaction effects including endosperm additive and dominant interaction effects, maternal additive and dominant interaction effect, and cytoplasmic interaction effects were found at most filling stages by both unconditional genetic analysis and conditional genetic analysis but cytoplasmic interaction effect in this study. Significant genotype $\times$ environment interaction effects also indicated that the sequential expression of genes from endosperm, cytoplasm and maternal genetic systems was also influenced by the environmental conditions. The genotype $\times$ environment interaction effect was the main cause of genetic differences across environments. It is necessary to consider the variation of rice quantitative traits in different environments because of the varied climatic conditions to which rice is exposed.

The genetic models and statistical analysis methods used in this experiment, which include genetic main effects and genotype $\times$ environment interaction effects, can be analyzed with only three generations, such as parents, $F_{1}$ and $F_{2}$ at different filling stages across a range of environments. Evidence of varying gene expression for leucine content during grain filling in rice serves to underscore the need to study developmental behavior of gene expression for important quantitative traits in other cereal crops.

\section{Acknowledgements}

The project was supported by the Science and Technology Office of Zhejiang Province, China (No. 2008C14071 and 011102471), Zhejiang Provincial Natural Science Foundation of China, 151 Foundations for the Talents of Zhejiang Province and the Exchange Program of Friendship between Zhejiang Government of China and Fukui of Japan.

\section{REFERENCES}

[1] W. Q. Dong, S. H. Shi and Y. J. Dong, "Breeding and Utility of a New Hybrid Rice Xieyou9516,” Zhejiang Agricultural Science, Vol. 5, No. 5, 1999, pp. 211-213.

[2] J. S. Chauhan, V. S. Chauhan and S. B. Lodh, "Environment Influence on Genetic Parameters of Quality Component in Rainfed Upland Rice," Indian Journal of Agricultural Science, Vol. 62, No. 5, 1992, pp. 773-775.
[3] K. F. Osato, Y. Hamachi and Y. Matsue, "Genotype $x$ Environment Interaction of Palatability in Rice,” Japanese Journal of Crop Science, Vol. 65, No. 5, 1996, pp. 585-589.

[4] C. H. Shi, J. M. Xue, Y. G. Yu, X. E. Yang and J. Zhu, "Analysis of Genetic Effects for Nutrient Quality Traits in Indica Rice,” Theoretical and Applied Genetics, Vol. 92, No. 8, 1996, pp. 1099-1102.

[5] C. H. Shi, J. Zhu, X. E. Yang, Y. G. Yu and J. G. Wu, "Genetic Analysis for Protein Content in Indica Rice," Euphytica, Vol. 107, No. 2, 1999, pp. 135-140.

[6] Y. F. Tan, J. X. Li and C. G. Xu, "Genetic Bases of Appearance Quality of Rice Grains in Shanyou 63, an Elite Rice Hybrid," Theoretical and Applied Genetics, Vol. 101, No. 5-6, 2000, pp. 823-829.

[7] C. W. Xu, A. H. Zhang and Q. S. Zhu, "Genetic Analysis of Quality Traits in Rice Crosses between Indica and Japonica,” Acta Agronomica Sinica, Vol. 22, No. 5, 1996, pp. 530-534.

[8] J. Zhu, "Analysis of Conditional Genetic Effects and Variance Components in Developmental Genetics,” Genetics, Vol. 141, No. 4, 1995, pp. 1633-1639.

[9] C. H. Shi, J. G. Wu and P. Wu, “A Developmental Behavior of Gene Expression for Brown Rice Thickness under Different Environments,” Genesis, Vol. 33, No. 4, 2002, pp. 185-190.

[10] C. H. Shi, J. G. Wu, X. B. Lou, J. Zhu and P. Wu, "Genetic Analysis of Transparency and Chalkiness Area at Different Filling Stages of Rice (Oryza sativa L.),” Field Crops Research, Vol. 76, No. 1, 2002, pp. 1-9.

[11] C. H. Shi, J. G. Wu, X. M. Zhang and P. Wu, “Developmental Analysis on Genetic Behavior of Brown Rice Recovery in Indica Rice across Environments,” Plant Science, Vol. 163, No. 3, 2002, pp. 555-561.

[12] J. G. Wu, C. H. Shi and X. M Zhang, "Estimating the Amino Acid Composition in the Milled Rice Powder by Near-Infrared Reflectance Spectroscopy,” Field Crops Research, Vol. 75, No. 1, 2002, pp. 1-7.

[13] J. Zhu and B. S. Weir, “Analysis of Cytoplasm and Maternal Effects: II. Genetic Models for Triploid EndospeRms,” Theoretical and Applied Genetics, Vol. 89, No. 2-3, 1994, pp. 160-166.

[14] R. G. Miller, “The Jackknife- A review,” Biometrika, Vol. 61, No. 1, 1974, pp. 1-15.

[15] J. Zhu and B. S. Weir, "Diallel analysis for sex-linked and maternal effects," Theoretical and Applied Genetics, Vol. 92, No. 1, 1996, pp. 1-9. 